\title{
Synthetic Collagen Fascicles for the Regeneration of Tendon Tissue
}

\author{
S.J. Kew \\ J. H. Gwynne \\ D. Enea \\ R. Brookes \\ N. Rushton \\ S. M. Best \\ R. E. Cameron
}

\begin{abstract}
The structure of an ideal scaffold for tendon regeneration must be designed to provide a mechanical, structural and chemotactic microenvironment for native cellular activity to synthesise functional (i.e. load bearing) tissue. Collagen fibre scaffolds for this application have shown some promise to date, although the microstructural control required to mimic the native tendon environment has yet to be achieved allowing for minimal control of critical in vivo properties such as degradation rate and mass transport. In this report we describe the fabrication of a novel multi-fibre collagen fascicle structure, based on type-I collagen with failure stress of $25-49 \mathrm{MPa}$, approximating the strength and structure of native tendon tissue. We demonstrate a microscopic fabrication process based on the automated assembly of type-I collagen fibres with the ability to produce a controllable fascicle-like, structural motif allowing variable numbers of fibres per fascicle. We have confirmed that the resulting post-fabrication type-I collagen structure retains the essential phase behaviour, alignment and spectral characteristics of aligned native type-I collagen. We have also shown that both ovine tendon fibroblasts and human white blood cells in whole blood readily infiltrate the matrix on a macroscopic scale and that these cells adhere to the fibre surface after seven days in culture. The study has indicated that the synthetic collagen fascicle (SCF) system may be a suitable biomaterial scaffold to provide a rationally-designed implantable matrix material to mediate tendon repair and regeneration.
\end{abstract}

\section{Introduction}

Regeneration of damaged tendon tissue remains an underserved medical need for the field of orthopaedics. These highly specialised and aligned tissues provide the structural support necessary in order to maintain appropriate biomechanical function in all joints of the human body. The properties of ligament and tendon vary substantially by anatomical location, with concomitant variation in structure and configuration. However, an invariant feature of these tissues is that they are substantially oriented type-I collagen fibre structures, providing load bearing and force transfer parallel to the direction of orientation. Once damaged, tendons and ligaments lose their ability to protect the joint from biomechanical loading patterns which can damage articular cartilage and may expedite the onset of degenerative changes such as osteoarthritis [1]. Furthermore, the loss of function and pain associated with injuries to tendon carries a significant societal burden via patient morbidity, notably in the Achilles 
[2] and shoulder rotator cuff tendons [3]. Current approaches to the surgical treatment of tendon injuries rely on fixation devices such as bone-anchoring sutures and biomaterial grafting in combination with various suture techniques to re-attach tissues and restore function to the damaged area. However, bone anchors and (bio)synthetic grafting are limited to providing a purely mechanical solution to what is often a biological problem, with degenerated or ruptured tendon tissue often found either prior to, or as a result of, a tendon injury [4]. However, autografting and muscular transfers are limited by a noticeable morbidity, and allografting may cause immunoreactions and spread infective diseases. Hence structural repairs often fail: for example in the case of massive rotator cuff repairs an estimated $13-68 \%$ will re-rupture [5 and references therein]. Therefore, the ability to provide a biological augmentation, which enhances the quality of the repaired tendon tissue in concert with the structural repair, is a promising methodology by which to reduce the significant problem of longevity in the current repair modalities. A multitude of approaches have been proposed towards the overall goal of regenerating tendon tissue, with combinations of biological species such as growth factors, stem cells, blood concentrates both with and without biomaterials all attracting significant current research interest.

Clinical attempts to provide a biomaterial scaffold that enables the recapitulation of tendon tissue have, to date, focussed on use of decellularised extra-cellular matrix materials (ECM) [6]. However, these materials are unlike the tissues they aim to regenerate, being non-axially oriented and retaining allogenic or xenogenic epitopes [7] and furthermore, are mechanically weaker than human tendon or ligament tissue. Few pre-clinical studies have shown significant performance advantages compared to non-augmented controls and some clinical studies have been abandoned due to post-operative complications [8]. Various nondegradable polymer fibre systems have been investigated as mechanical supports for soft tissue. However, their usage has also been limited owing to long-term complications associated with mechanical degradation and production of wear debris, which ultimately have resulted in numerous failures such as, in the anterior cruciate ligament (ACL) site [9]. Hence there remains a significant unmet clinical need for a degradable biomaterial that can be shown to mediate the replacement of damaged tendon with native-type tissue that can play a structural role in support of the joint.

Reconstituted type-I collagen fibres have attracted interest as scaffolds for the regeneration of tendon tissue owing to their structural similarity to native tissues [10]. Such collagen fibre scaffolds have been used to repair tendon tissue in vivo in a number of systems, including rabbit Achilles tendon and anterior cruciate ligament [11]. The initial mechanical properties of the scaffolds are of importance for two reasons, firstly for primary surgical fixation and secondly to provide mechanical substitution or augmentation (i.e. functional tissue engineering [12]). In the case of tendon tissue, the substitutive requirement is substantially more demanding than the augmentative, with tendon tissue substitution typically requiring break stresses of $>50 \mathrm{MPa}$ [13], whereas augmentation can be achieved with break stresses an order of magnitude lower.

The crosslinking chemistries for collagen biomaterials have been extensively investigated and a recent study has compared the mechanical properties achieved by numerous approaches, finding significant deviation from previously reported values [14]. This deviation highlights the effect of collagen source, preparation/purification conditions and 
the various process steps such as post-extrusion stretching [15]. Fibrillogenesis may also have a significant effect on the final mechanical properties and biological properties of collagen fibres [16,17]. Furthermore, crosslinking conditions and associated denaturation of the collagen structure have a significant effect of the longevity of mechanical properties in proteolytic biological environments [18]. Collagen fibres bioscaffolds have yet to find clinical application, possibly due to the limited control over critical in vivo performance characteristics, such as degradation rate mechanical properties and mass transport that enables tailoring for particular tissues. We have begun addressing this limitation by designing a novel biomaterial scaffold which replicates the structure of tendon tissue [19] with a biomimetic synthetic collagen fascicle (SCF) structure. We hypothesise that this structure will provide the necessary structural and chemotactic signals to cells infiltrating the scaffold based not only on the structure of the constituent fibre but also on the fascicle assembly itself. Furthermore, we postulate that the fascicle structure per se will provide a templating effect of the synthesis of new matrix that will provide long-term benefit in the deposition of neo-tendon tissue.

In this report we describe a microscopic assembly technique to fabricate a synthetic collagen fascicle, which represents a novel approach to creating a tendon-mimetic type-I collagen fibre scaffold. We selected carbodiimide crosslinking chemistry for this study based on its known biocompatibility and moderate strengthening effect. In addition, we added a secondary diepoxide crosslinker, which was used to further enhance mechanical properties by utilising non-proximate nucleophilic groups that may not have been accessible to the zero-length carbodiimide coupling reaction. As has previously been reported by our group [20] we propose that control over mechanical strength, degradation rate and chemotactic signalling of the scaffold can be used to rationally design a synthetic collagen fascicle that provides an ideal microenvironment for tendon regeneration.

\section{Materials and Methods}

\subsection{Synthetic Collagen Fascicle Fabrication}

Collagen Preparation: Acid swollen type-I collagen from bovine corium (Devro Medical, Glasgow, UK) was dispersed at $6 \mathrm{mg} \mathrm{mL}^{-1}$ in $2 \mathrm{mM} \mathrm{HCl}$ for at least 15 hours, prior to being blended for four minutes on full power using a blender (Kenwood, Multi-pro). The gel temperature did not exceed $25^{\circ} \mathrm{C}$. The collagen gel was then degassed at 20 mTorr and stored in $30 \mathrm{~mL}$ syringes at $4^{\circ} \mathrm{C}$ for up to two weeks. Collagen Fibre Extrusion: The collagen gel was extruded from a $0.51 \mathrm{~mm}$ microbore tubing at $0.3 \mathrm{~mL} \mathrm{~min}{ }^{-1}$ using a syringe pump (Chemxy fusion 200, KR Analytical, Cheshire, UK) into a stainless steel bath containing "fibre formation buffer" (FFB), which consisted of phosphate buffered saline (PBS) and 20\% w/v poly(ethylene glycol) (PEG) $\mathrm{M}_{\mathrm{w}} \sim 8000$ (Sigma-Aldrich, Dorset, UK) at $37^{\circ} \mathrm{C}$. The fibre was incubated for at least five minutes in FFB to allow coagulation and partial fibrillogenesis to occur [21]. Synthetic Collagen Fascicle Fabrication: The fibre was then wound onto a spool using a custom-built automated system that utilised a rotating and translating spool (Figure 1). To produce a synthetic collagen fascicle scaffold, collagen/PEG fibres were 
wound in a multiple-fibre over-layer structure to produce an assembly, comprising a controlled number of separate strands. This allowed the fibre assembly to be incubated and dried overnight, thus allowing the nascent fibre to continue the fibrillogenesis process at increasing PEG concentrations during the drying phase. The final dried assembly was a collagen/PEG composite (confirmed by FTIR). The fascicle assembly was air dried overnight to adhere the fascicle structures, which remained assembled for the entire fabrication process. The individual fibres were visible in the final dried assembly. Crosslinking: The dried collagen/PEG composite was simultaneously crosslinked and washed on the spool in an $80 \%$ acetone/water solution, which performed two functions: firstly to crosslink the collagen component and secondly to remove the PEG component. The assembly was then crosslinked in either: (i) A solution of $25 \mathrm{mM}$ EDC and $12.5 \mathrm{mM}$ NHS in $80 \%$ acetone/PBS for two hours or (ii) A solution of $25 \mathrm{mM}$ EDC and $12.5 \mathrm{mM}$ NHS in $80 \%$ acetone/PBS for two hours followed by incubation in a solution of $1 \%(\mathrm{v} / \mathrm{v})$ ethyleneglycol diglycidylether (EGDE) in PBS for five days. Final washing: After crosslinking, the assembly was washed on the spool with PBS for $2 \times 30$ minutes and ultra-high purity water $(R>18 \mathrm{M} \Omega)$ for $2 \times 30$ minutes. The hydrated and crosslinked assembly was then air dried overnight, before being manually detached from the spool. Removal of PEG was confirmed via both differential scanning calorimetry and Fourier transform infra red spectroscopy in comparison with unwashed controls (data not shown). The dried assembly was then sterilised using $\gamma$ irradiation at $25 \mathrm{kGy}$.

\subsection{Physical Characterisation}

Mechanical Testing: The extruded synthetic collagen fascicles assemblies were hydrated in PBS overnight at $22^{\circ} \mathrm{C}$ and mechanically tested using an Instron 3343 Universal testing machine (Instron, High Wycombe, UK) with flat face contact grips. The scaffolds were tested at $10 \mathrm{~mm} \mathrm{~min} \mathrm{~m}^{-1}$ and all scaffolds failed within the body of the implant and not at the grips. The scaffolds were measured using a micrometer to determine the grip to grip distance. The individual fibres were tested using fibre-specific grips (Instron) and were hydrated in PBS overnight prior to testing. Any fibres that failed at the grips were excluded from the sample. The individual fibres were obtained from the synthetic collagen fascicles. Mechanical testing was performed immediately after immersion in PBS to minimise the effect of drying on the fibre mechanical properties. Fibre diameter was determined with the fibres hydrated in a small volume $(\sim 100 \mu \mathrm{L})$ of PBS using a calibrated optical microscope, 3-5 diameters were measured per fibre and averaged. Polarised Optical Microscopy: Microscopy was performed using collagen fibres and scaffolds which were hydrated in PBS overnight and placed on a glass cover slip. The microscope used was a L2000B HTG (GT Vision, Suffolk, UK). Differential Scanning Calorimetry (DSC): DSC was carried out using a TA Instruments Q2000 DSC. Dry samples (with mass between 2 and $5 \mathrm{mg}$ ) were placed in aluminium pans (not hermetically sealed). The samples were equilibrated at $5^{\circ} \mathrm{C}$ and then heated at $10^{\circ} \mathrm{C} / \mathrm{min}$ to $250^{\circ} \mathrm{C}$. Fourier Transform Infra-red Spectroscopy (FTIR). FTIR studies were carried out using a Bruker Tensor 27 Infrared Spectrometer with a diamond ATR crystal. Scans were carried out from 4000 to $550 \mathrm{~cm}^{-1}$, with a resolution of $8 \mathrm{~cm}^{-1}$, and 16 scans were averaged to produce the spectra. Small Angle X-ray Scattering (SAXS). Scans were carried out using a Bruker Nanostar, with a sample-to-detector distance of $106 \mathrm{~cm}$. 
Scans needed to be carried out for 10000 seconds, due to low intensities. A background correction was carried out, taking into account the sample transmission (measured using a glassy carbon sample). Scanning electron microscopy (SEM). Synthetic collagen fascicles were sputter-coated with a thin layer of gold (20 mA for 2 minutes) and examined using a JEOL 820 SEM.

\subsection{Biological Characterisation}

Tendon fibroblast Cell Culture. Patellar tendon tissue was harvested in a sterile fashion from sheep and washed 3 times in fresh DMEM supplemented with $10 \%$ fetal calf serum (FCS), $2 \mathrm{mM}$ glutamine, $100 \mathrm{IU} / \mathrm{ml}$ penicillin, $100 \mathrm{ug} / \mathrm{mL}$ streptomycin and $10 \mathrm{ug} / \mathrm{mL}$ gentamicin (complete DMEM). The tissue was dissected and finely minced and approximately $5 \mathrm{~g}$ of wet weight of tissue was put into a $175 \mathrm{~mL}$ flask, to which was added $50 \mathrm{~mL} \mathrm{0.2 \%} \mathrm{collagenase} \mathrm{1A} \mathrm{(Sigma)} \mathrm{in} \mathrm{supplemented} \mathrm{DMEM.} \mathrm{The} \mathrm{tissue} \mathrm{was} \mathrm{incubated} \mathrm{at}$ $37^{\circ} \mathrm{C}$ with shaking for 2-3 hours, by which time most of the tendon had dissolved into a thick cell suspension. This suspension was spun at $1500 \mathrm{rpm}$ for 5 minutes and the cells washed twice in fresh medium before plating in $175 \mathrm{~cm}^{3}$ flasks. Cells were allowed to adhere for 2-3 days, after which the medium was changed. Cells were trypsinized, re-suspended and re-plated in $175 \mathrm{~cm}^{3}$ flasks in supplemented DMEM, grown to confluence and used at the third passage. Blood Infiltration: About 100 six-fibre synthetic collagen fascicles were extruded, dried, crosslinked, placed in parallel and sutured with 1-0 nylon thread every 1 $\mathrm{cm}$ and then cut to size. Bundles of fibres $(1 \mathrm{~cm}$ long) were vertically placed under tension into a sterilized cryosectioning cube. Samples were rinsed three times in sterile PBS. A blood sample was taken from a healthy human donor and immediately poured into the cryosectioning cubes without anticoagulant. $3 \mathrm{~mL}$ blood was poured into each cube so as to cover completely the bundle. Samples were then placed into the incubator for $24 \mathrm{~h}$. After 24 $h$, the day 1 samples were harvested and the day 3 and day 7 samples were aspirated to remove the serum which had separated from the blood clot, and complete DMEM was added so as to completely cover the bundles. The medium was renewed on the third day. On the day of the harvest, the serum (day 1) or medium (day 3 and day 7) was aspirated and OCT compound was poured into the cryosectioning cubes. As the clot was contracted onto the bundle at the centre of the cube, the OCT distributed on the periphery of the cube adhering to the plastic walls. Cubes were then placed into a $-80^{\circ} \mathrm{C}$ freezer until the day of cutting. The cryo-blocks were cut into $7 \mu \mathrm{m}$ thick sections. These were placed on Poly-Prep slides (Sigma) and quickly air dried, fixed in ice cold methanol for 10 minutes, air dried again and placed in Giemsa stain (Sigma) (1:10 dilution in distilled water) for 40 minutes. Slides were visualised under optical microscopy (Leitz Laborlux 12) using digital image acquisition. Scanning Electron Microscopy (SEM): The attachment of ovine tendon fibroblast cells was also evaluated using SEM. Collagen fibres (NHS/EDC, NHS/EDC+EGDE) were hydrated for one hour in PBS and seeded with tendon fibroblasts at a seeding density of $10^{4}$ cells in $200 \mu \mathrm{L}$ of complete DMEM. Cells were then cultivated for 24 hours and fixed in $4 \%$ glutaraldehyde in $0.1 \mathrm{M}$ pipes buffer at $\mathrm{pH} 7.4$ for 72 hours at $4{ }^{\circ} \mathrm{C}$. They were rinsed in deionised water and treated with $1 \%$ osmium ferricyanide at $4{ }^{\circ} \mathrm{C}$ for 18 hours. They were rinsed in deionised water and treated with $2 \%$ uranyl acetate in $0.05 \mathrm{M}$ maleate buffer at $\mathrm{pH} 5.5$ for 18 hours at $4{ }^{\circ} \mathrm{C}$. They were again rinsed in deionised water and dehydrated in an ascending series of ethanolic solutions from $70 \%$ to $100 \%$. They were placed in a Polaron critical point dryer (Quorum/Emitech, UK) where the ethanol was 
replaced with liquid $\mathrm{CO}_{2}$ which was heated to $37^{\circ} \mathrm{C}$ where it changed state to its gaseous phase. The $\mathrm{CO}_{2}$ gas was vented and the samples were glued to SEM stubs with colloidal silver. The stubs were coated with $20 \mathrm{~nm}$ of gold in a Quorum/Emitech K575X sputter coater. They were viewed in an FEL-Philips XL30 FEG-SEM operated at $5 \mathrm{kV}$.

\section{Results and Discussion}

\subsection{Microscopic Structure: Assembly of Synthetic Collagen Fascicles (SCF)}

Extrusion of acidified collagen gels into a fibre forming buffer at neutral $\mathrm{pH}$ and physiological temperatures is a well-known methodology by which to produce oriented collagen fibres based on the spontaneous assembly of type-I collagen in solution [22 and references therein]. However, the ability to produce sufficient quantities of collagen fibres on a laboratory scale has consistently limited the development of these biomaterials. Furthermore, these systems are limited in providing a single fibre cross-sectional motif and key parameters such as interstitial dimensions and specific surface area of the final scaffold cannot be readily controlled. It is known that the structural dimensions for porous materials strongly impact upon the biological function of open-cell porous biomaterials [23]. Therefore, a materials fabrication methodology that can control the equivalent structural parameter for fibre assemblies (i.e. inter/intra fibre packing) is anticipated to be useful for the control of implant degradation, cellular infiltration and metabolic transport. We calculated the specific surface areas for a variety of fascicle configurations (Figure 2) using an idealised fibre morphology and show how the specific surface area can be varied by changing the number of fibres per fascicle. We were able to conveniently change the number of fibres per fascicle by changing the number of extrusion tubes between two and twelve and we used a twelve fibre fascicle for this study as a model system.

The assembly of synthetic collagen fascicles was achieved by overlaying hydrated collagen fibres into a multithread array whilst hydrated with PBS and PEG. The presence of PEG provided a means by which to retain the circular fibre section of each structural unit of the multi-thread assembly as it was dried; this is used to define the morphology of the fibre with respect to the adjacent fibres. Furthermore it enabled the retention of a circular fibre section, thus presenting surface curvature commensurate with that of the constituent fibres (see Figure 3). We have found that PEG is necessary during drying to retain the constituent fibre structure, without which the collagen fibres intermingle to form an unstructured mass during drying. We evaluated a number of fibre formation buffers (TES, TRIS) and PEG concentrations $(5 \%, 10 \%)$ and found that $20 \%$ PEG / PBS was necessary to provide sufficient wet strength for the fibre to be wound using our translating spool set-up. The temperature of the fibre formation buffer was set at $37^{\circ} \mathrm{C}$ to maximise fibrillogenesis: at room temperature $\left(22^{\circ} \mathrm{C}\right)$, the wet fibre was noticeably weaker, indicating a less well assembled

structure. The translating rotating spool was used to achieve a defined number of fibre overlays per fascicle, thus enabling control over the number of fibres present in each unit. 
For this study we selected twelve fibres per fascicle as our model system for physical characterisation; however this number can be conveniently tailored via the assembly process (shown in Figure 1) from two to $>100$ fibres per fascicle. The dimensions of the hydrated fascicle assembly were selected at twelve fibres per fascicle to be microscopically similar to that observed for dissected ovine patellar tendon tissue as shown in Figure4. SEM and polarised optical microscopy images of the fascicle assemblies are shown in Figures 3 and 4. The polarised optical microscopy indicated axial collagen fibre alignment by appearing bright under crossed polarising filters. The micrographs were compared with partially dissected ovine patellar tendon (Figure $4 \mathrm{C}$ ) to show that the structure was qualitatively similar, although the optically observable banding patterns of native type-I collagen assembly were not observed for the SCF scaffolds, indicating that extensive native-type fibrillogenesis was not occurring as has been recently observed for fibres incubated at neutral $\mathrm{pH}$ and $37^{\circ} \mathrm{C}$ at time periods $>12$ hours [17]. The lack of the native d-banding in our scaffolds indicated that there may have been insufficient time for the self-assembly process to occur and/or that the mobility required was absent due to either the type or amount of pre-assembly crosslinking in the acidified collagen system.

\subsection{Mechanical Behaviour}

\begin{tabular}{|c|c|c|c|c|c|}
\hline Crosslinking & $\begin{array}{l}\text { Failure } \\
\text { Stress } \\
\text { MPa }\end{array}$ & $\begin{array}{l}\text { Failure } \\
\text { Strain / \% }\end{array}$ & $\begin{array}{l}\text { Elastic modulus } \\
\text { at } 5-7 \% \text { strain / } \\
\mathrm{MPa}\end{array}$ & $\begin{array}{l}\text { Tested } \\
\text { Length } \\
\text { (grip to } \\
\text { grip) } / \mathrm{mm}\end{array}$ & $\begin{array}{l}\text { Diameter } \\
/ \mu \mathrm{m}\end{array}$ \\
\hline $\begin{array}{l}\text { (i) Fibre } \quad(\mathbf{n}=5): \\
\text { NHS/EDC }\end{array}$ & $25.1( \pm 9.2)$ & $13.5( \pm 4.4)$ & $183.5( \pm 76.2)$ & $17.6( \pm 3.2)$ & $58.3( \pm 5.2)$ \\
\hline $\begin{array}{l}\text { (ii) Fibre } \quad(\mathbf{n}=\mathbf{5}) \text { : } \\
\text { NHS/EDC + EGDE }\end{array}$ & $49.0( \pm 22.1)$ & $17.3( \pm 5.6)$ & $276.8( \pm 168.4)$ & $11.7( \pm 1.7)$ & $59.2( \pm 13.3)$ \\
\hline $\begin{array}{ll}\text { (iii) SCF } & (\mathbf{n}=4): \\
\text { NHS/EDC } & \end{array}$ & $4.6( \pm 2.0)$ & $27.2( \pm 12.0)$ & $27.2( \pm 13.2)$ & $32.0( \pm 3.2)$ & $4240( \pm 560)$ \\
\hline $\begin{array}{l}\text { (iv) SCF } \quad(\mathbf{n}=5): \\
\text { NHS/EDC + EDGE }\end{array}$ & $16.9( \pm 2.6)$ & $26.6( \pm 2.2)$ & $76.0( \pm 8.9)$ & $21.5( \pm 2.9)$ & $3420( \pm 230)$ \\
\hline
\end{tabular}

Table 1: Mechanical properties of crosslinked collagen fibres used as components of the synthetic collagen fascicles (i/ii) and twelve fibre synthetic collagen fascicles (12 fibres per fascicle) (iii/iv); one standard deviation shown as an indication of precision.

As shown in Table 1, the effect of the EGDE crosslinking was to increase the failure stress for the constituent fibre by approximately two-fold to $48 \mathrm{MPa}$ compared with $25 \mathrm{MPa}$ for NHS/EDC alone such that the crosslinked collagen fibres have comparable mechanical strength to human anterior cruciate ligament tissue (mean failure stress $=13.3-37.8 \mathrm{MPa}$ ) [24] or rotator cuff supraspinatus tendon (mean failure stress $=4.1-16.5 \mathrm{MPa}$ ) [35] although not Achilles tendon (mean failure stress $=71 \mathrm{MPa}$ ) [13]. The failure stress for ovine patellar tendon, upon which this scaffold design was modelled, was found to be 20.8( \pm 7.9$) \mathrm{MPa}$ and failure strain was $19.7( \pm 3.7) \%$, a detailed description of this testing will be published elsewhere. 
The SCF scaffold produced using either fibre type was significantly weaker than the fibres alone $(\mathrm{P}<0.05)$, an effect that has been reported previously by Gentleman et al. [25] for collagen gel - fibre composites. The origin of this effect is not entirely clear, although we propose that the ostensible weakening of the SCFs may be associated with the mechanical test method using face grips that are unable to transfer equal load to each fibre constituent. This is supported by the observed increase in strain to failure for the scaffolds, which is likely to be the result of inter-fascicle slippage; the apparent modulus of the SCF scaffold was also reduced. Hence the overall mechanical properties of these constructs would not be sufficient to replace the mechanical function of tendon tissue in the shoulder or ankle, although the constituent fibres were of comparable strength.

Fabrication of collagen bioscaffolds with mechanical properties that match those of actual tendon has remained a significant challenge and despite previous reports of fibre mechanical properties $[10,26]$ that are comparable with tendon, the mechanical properties of fibre assemblies are reported to be approximately a quarter of the constituent fibres [27, 28]. This discrepancy is presumably a result of the inability of the final scaffold to support loading of all fibres; however, it does mean that collagen fibre scaffolds are likely to be mechanically weaker than the tendon or ligament they replace when surgically fixated. Thus the practical requirement for these biomaterials as augmentative devices is that they can be attached via a surgical fixation that confers sub-failure loading to the biomaterial. This is achieved using ECM tendon repair patches where the mechanical properties of the final constructs are suitable for suture fixation to tendon tissue, without full load-bearing; ECM tendon reinforcement patches fail in the stress range ca. $5-10 \mathrm{~Pa}$ at $27-229 \mathrm{~N}$ force $[29,30]$. Thus the mechanical strength required for fixation in a human tendon repair would also be achieved using these SCF bioscaffolds, which exhibited failure stresses that are comparable in magnitude at 4.6-16.9 MPa.

\subsection{Structural Characterisation}

The physical structure of the synthetic collagen fascicle was characterised using differential scanning calorimetry (DSC), fourier transform infra-red spectroscopy (FTIR) and small angle x-ray scattering (SAXS). 2D small angle x-ray diffraction patterns for the two types of SCF are shown in figure 5 and confirm the presence of alignment within the collagen fibres parallel to the extrusion direction (which is horizontal relative to the images shown). The peaks in the horizontal direction correspond to high-orders of diffraction from the $64 \mathrm{~nm}$ repeat along the collagen fibres, whereas the peaks in the vertical direction are related to the inter-fibril packing distance. Samples crosslinked using the two different methods showed similar degrees of alignment.

DSC results are also shown in figure 5, and indicate the absence of any residual crystalline PEG within the SCF structure, since this would be seen as a characteristic sharp peak (Figure 5). The broad peak at lower temperatures is caused by denaturation of the triple-helical collagen structure and is dependent on the water content $[31,32]$. The second peak, seen at 
temperatures of approximately $220^{\circ} \mathrm{C}$ is likely to be due to loss of the secondary collagen structure and occurs at a similar temperature for both types of SCF.

FTIR-ATR spectra for the two SCFs are shown in figure 6; this technique can be used to study the extent of collagen denaturation using two mid-IR peaks: the peak at $1235 \mathrm{~cm}^{-1}$, which is an amide III band and is sensitive to the triple-helix content of collagen, and the peak at $1450 \mathrm{~cm}^{-1}$, which corresponds to $\mathrm{CH}_{2}$ and $\mathrm{CH}_{3}$ deformation and is invariant with changes in extent of denaturation [34]. The higher the peak height ratio $\mathrm{A}\left(1235 \mathrm{~cm}^{-1}\right): \mathrm{A}(1450$ $\mathrm{cm}^{-1}$ ), the greater the triple-helix content; he peak height ratio for unprocessed acid swollen collagen was found to be $1.13 \pm 0.01$ whereas that for unprocessed gelatine (Sigma Aldrich) was found to be $0.95 \pm 0.01$. The SCFs crosslinked using both chemistries had peak height ratios between these two values (1.02 \pm 0.01 using NHS/EDC and 1.02 \pm 0.01 using NHS/EDC + EGDE), which indicates that partial denaturation of the collagen structure had occurred during crosslinking and/or extrusion processing. Partial denaturation of the collagen structure may have a detrimental effect on the mechanical properties of the fibres owing to the reduction of concerted load bearing via fiber interdigitation however, it was found that the cross-linking chemistry had a dominant effect on mechanical properties. Therefore, it was concluded that effective crosslinking was a key component for achieving sufficient mechanical strength.

\subsection{In vitro Biological properties of the Synthetic Collagen Fascicle}

\subsubsection{Infiltration of SCFs with Human blood}

There are several studies in literature focused on the in vivo implantation of bundles of reconstituted collagen fibres [22 and reference therein]. In some cases, to ease the surgical delivery of the scaffold, bundles were made by manually aligning collagen fibres cast in collagen gel [11, 28]. However, this system may block the early infiltration of cells into the interior of the implant. Cavallaro et al. implanted a bundle made by a continuous thread of extruded collagen arranged to form a closed loop into a canine knee [33]. However, this study was limited in scope with only two cases at a single time point reported, with the anterior cruciate ligament as the target tissue. The system of open collagen fibre structures has not yet been investigated in the context of tendon repair and may provide a promising strategy to scaffold repair of tendon tissue. In particular, the possibility of an open array of synthetic collagen fascicles is attractive because it is hypothesised that it may ease the immediate cellular and vascular ingrowth and therefore it may improve the repair capabilities of the implant. A key element of this biomaterial design was to provide the ability to control specific surface area and the interstices available for neo-tissue formation such that a biomimetic micro-structure is produced. Furthermore, the microscale similarity between the SCF implant and the native tissues may provide a structural cue to the body to generate a native organisation of new vascularity and neo-tendon tissue in vivo.

As a preliminary model to evaluate the ability of the dry implant to be infiltrated with blood, the SCF implants were hydrated using human whole blood. The blood-hydrated implant was then imaged histologically in cross-section. It was found that at lower magnification (Figure 8A) it was possible to observe a great number of individual SCF 
strands. These fascicles were independent from one another and they were freely permeated by white blood cells (WBC) and blood components. At higher magnification (figures 7), WBCs were observed to adhere to single collagen fibres at 1,3 and 7 days after seeding. No differences in cellularity or in other morphological aspects were observed between the different time-points. Collagen fibres were shown to have mostly round or elongated cross sectional areas and to contain some defects such as voids between the fibers, indicative of incomplete fascicle packing. As anticipated from the fibre close-packing structures proposed (Figure 2), irregular surface features consistent with a faceted fibre arrangement were observed although physical deformation most likely causes the significant deviations from the idealized geometric representations of Figure 2.

\subsubsection{Initial Stage Interactions of the SCF with Tendon fibroblasts}

The purpose of this biomaterial design was to provide a controllable structure that enables the attachment and proliferation of tendon fibroblasts with the overall aim that they deposit neo-tendon tissue. Initially, this regenerative process would be expected to deposit tissue within the inter-fascicle voids and as the implant degrades, the SCF structures are anticipated to degrade with concomitant tissue deposition. To examine this possibility in the initial stages of healing, we evaluated the process of seeding the scaffold with ovine tendon fibroblasts and the morphological features of the scaffold and cells that had attached to the structure up to seven days in culture. It was found that both crosslinking chemistries were suitable for tendon fibroblast attachment; however, as shown in figure 8, the NHS/EDC chemistry was noticeably more amenable to cellular spreading, with tendon fibroblasts assuming a flattened or elongated phenotype. It was found that a significant number of tendon fibroblasts that attached to the NHS/EDC + EDGE crosslinked fibres were spheroidal in morphology, possibly indicating suboptimal interactions with the matrix. The interactions between these crosslinked fibres and ovine tendon fibroblasts have been investigated in detail by our group [20] and these morphological findings further supported the biochemical data which indicates reduced matrix deposition onto the NHS/EDC + EGDE crosslinked collagen matrix compared with NHS/EDC alone. In addition, we reported expression levels of soluble the tendon tissue markers Type-1/Tenascin results, which also indicated that the NHS/EDC crosslinked structures were more compatible with ovine tenocyte cells than NHS/EDC and EGDE.

\section{Conclusions}

In this study we have described a novel method for the synthetic assembly of type-I collagen fibres to form a pseudo-fascicle structure, which mimics the microscopic structure of native tendon tissue. The purpose of the study was to develop a method of assembly that provides a structure that not only mimics the native composition and orientation of tendon but also its microscopic structure. The scaffold structure that was developed may enable the tailoring of key microscopic parameters including the specific surface area and dimensions of interunit interstices. Thus, this method demonstrates the possibility of controlling key parameters for in vivo use such as degradation rate, cellular infiltration and diffusion of nutrients in and out of the structure. In this report we have showed that the physical and 
biological properties of the type-I collagen structure indicate suitability for use in tendon tissue engineering. We have developed a novel methodology to achieve modulation of both microscopic architecture and the properties conferred by crosslinking chemistry, such as mechanical properties chemotactic signalling and degradation rate. We plan to further evaluate these materials in biological environments with the overall aim of developing a programmable type-I collagen fibre architecture that can achieve the regeneration of damaged tendon tissue.

\section{Acknowledgements}

S.J.K., J.H.G., D.E., R.B., N.R., S.M.B. and R.E.C. would like to thank the Technology Strategy Board for support under Grant No. TP/8/BIO/6/I/Q0052K. We would also like to thank Timothy Mead and Oliver Ferguson for assistance in construction of the extrusion equipment.

\section{Disclosure}

This work was supported in part by Tigenix Ltd, Cambridge, UK. 


\section{Figures}

Figure 1: Schematic for the production equipment used to produce a synthetic collagen fascicle with various numbers of constituent collagen fibres possible based on control of the spool's translation and rotational speed.

Figure 2: Schematic of assembled SCF structures (in 2D axial plan view) showing inter-fibre packing arrangements that are possible using this assembly technique. Specific surface areas were calculated based on a 50 micrometer diameter fibre for various faceted hexagonal close packed fascicle assemblies. Inset schemes show proposed cubic inter-fascicle packing structure showing the enlarged interstices expected for the SCFs compared with the close-packed single fibre motif. Inset is a photograph of a SCF bundle.

Figure 3: Scanning electron microscopy (right) and dry optical microscopy (left) of synthetic collagen fascicles showing individual units of the synthetic collagen fascicle structure. The electron micrograph shows the multi-filament nature of the synthetic fascicles and that the constituent fibres retain the morphology of the individual units. No notable differences were observed between the samples crosslinked using either A/B: NHS/EDC or C/D: NHS/EDC + EGDE as the crosslinking system.

Figure 4: Polarised optical microscopy images, shown in three rows of representative micrographs, of dissected synthetic collagen fascicles crosslinked using A: NHS/EDC or B: NHS/EDC + EGDE and C: ovine patellar tendon tissue dissected to various microstructural levels. All samples were imaged after $>24$ hours incubation in PBS. The scale bar applies to all micrographs shown.

Figure 5: Small Angle X-ray scattering results (left) and Differential Scanning Calorimetry results (right) of the synthetic collagen fascicle crosslinked using either NHS/EDC (top) or NHS/EDC + EDGE (bottom). The DSC for PEG ( $\mathrm{n}=3$ ) alone is also shown superimposed, indicating lack of residual PEG in the final crosslinked SCF structures.

Figure 6: FTIR spectra of the synthetic collagen fascicle crosslinked using either NHS/EDC or NHS/EDC + EDGE. In addition, the spectra for the collagen raw material, prior to processing and crosslinking and the PEG used in the fibre formation buffer is shown for reference.

Figure 7: Histological appearance of the synthetic collagen fascicle bundles immersed in a blood clot after seven days in culture and sectioned perpendicular to the fibre axis. Blood was found to permeate the space between single fascicles freely and white blood cell (WBC) nuclei were observed adherent to the fibre surface. The whole SCF bundle is $4-5 \mathrm{~mm}$ is diameter, increasing magnifications were used $\mathrm{A}<\mathrm{B}<\mathrm{C}$. Inset are photographs of the SCF scaffold structure attached to the metal spring used to impose mechanical alignment and tension on the scaffold.

Figure 8: SEM images showing ovine tendon fibroblast cells adhered to the synthetic collagen fascicle biomaterial A: NHS/EDC crosslinking chemistry B: NHS/EDC + EDGE crosslinking chemistry. SCF structure was imaged after twenty four hours in cell culture (details in materials section). 


\section{References}

1. Feeney MS, O’Dowd J, Kay EW, Colville J. Glenohumeral articular cartilage changes in rotator cuff disease. J Shoulder Elbow Surg 2003; 12: 20-23

2. Maffulli N. Current Concepts Review - Rupture of the Achilles tendon. J Bone Joint Surg Am 1999; 81: 1019-1036

3. Longo UG, Berton A, Papapietro N, Maffulli N, Denaro V. Epidemiology, Genetics and Biological Factors of Rotator Cuff Tears. Med Sport Sci 2012; 57: 1-9

4. Sharma P, Maffulli N. Tendon Injury and Tendinopathy: Healing and Repair. J Bone Joint Surg Am 2005; 87: 187-202

5. Rotini R, Fini M, Giavaresi G, Marinelli A, Guerra E, Antonioli D, Castagna A, Giardino R. New perspectives in rotator cuff tendon regeneration: review of tissue engineered therapies. Chir Organi Mov (2008) 91:87-92

6. Badylak, SF. The extracellular matrix as a biologic scaffold material. Biomaterials 2007; 28: 3587-3593

7. Zheng MH, Chen J, Kirilak Y, Willers C, Xu J, Wood D. Porcine small intestine submucosa (SIS) is not an acellular collagenous matrix and contains porcine DNA: possible implications in human implantation. J Biomed Mater Res B Appl Biomater 2005; 73: 61-67

8. Walton JR, Bowman NK, Khatib Y, Linklater J, Murrell GAC. Restore orthobiologic implant: not recommended for augmentation of rotator cuff repairs. J Bone Joint Surg Am 2007; 89: 786-791.

9. Mody BS, Howard L, Harding ML, Parmar HV, Learmonth DJ. The ABC carbon and polyester prosthetic ligament for ACL-deficient knees. J Bone joint Surg 1993; 75B: 818-821

10. Kato YP, Christiansen DL, Hahn RA, Shieh SJ, Goldstein JD, Silver FH. Mechanical properties of collagen fibres: a comparison of reconstituted and rat tail tendon fibres. Biomaterials 1989; 10: 38-42

11. Dunn MG, Tria AJ, Kato YP, Bechler JR, Ochner RS, Zawadsky JP, Silver FH. Anterior cruciate ligament reconstruction using a composite collagenous prosthesis. Am J Sports Med 1992; 20: 507-515

12. Butler DL, Juncosa-Melvin N, Boivin GP, Galloway MT, Shearn JT, Gooch C, Awad $\mathrm{H}$. Functional tissue engineering for tendon repair: A multidisciplinary strategy using mesenchymal stem cells, bioscaffolds, and mechanical stimulation. J Orthop Res 2008; 26: 1-9

13. Wren TAL, Yerby SA, Beaupré, Carter DR. Mechanical properties of the human achilles tendon. Clin Biomech 2001; 16: 245-251

14. Zeugolis DI, Paul GR, Attenburrow G. Cross-linking of extruded collagen fibers: A biomimetic three-dimensional scaffold for tissue engineering applications. J Biomed Mater Res A 2009; 89A: 895-908

15. Pins GD, Christiansen DL, Patel R, Silver FH. Self-assembly of collagen fibers. Influence of fibrillar alignment and decorin on mechanical properties. Biophys J 1997; 73: $2164-2172$

16. Silver FH, Freeman JW, Seehra GP. Collagen self-assembly and the development of tendon mechanical properties. J Biomech 2003; 36: 1529-1553 
17. Caves JM, Kumar VA, Wen J, Cui W, Martinez A, Apkarian R, Coats JE, Berland K, Chaikof EL. Fibrillogenesis in continuously spun synthetic collagen fiber. J Biomed Mater Res 2010; 93B: 24-38

18. Weadock KS, Miller EJ, Keuffel EL, Dunn MG. Effect of physical crosslinking methods on collagen-fiber durability in proteolytic solutions. J Biomed Mater Res 1996; 32: 221-226

19. Kastelic J, Galeski A, Baer E. The Multicomposite Structure of Tendon. Connect Tissue Res 1978; 6: 11-23

20. Enea D, Henson F, Kew S, Wardale J, Getgood A, Brooks R, Rushton N. Extruded collagen fibres for tissue engineering applications: effect of crosslinking method on mechanical and biological properties. J Mater Sci Mater Med 2011; 22: 1569-1578

21. Zeugolis DI, Paul RG, Attenburrow G. Extruded collagen-polyethylene glycol fibers for tissue engineering applications. J Biomed Mater Res A 2008; 85B: 343-352

22. Kew SJ, Gwynne JH, Enea D, Abu-Rub M, Pandit A, Zeugolis D, Brooks RA, Rushton $\mathrm{N}$, Best SM, Cameron RE. Regeneration and repair of tendon and ligament tissue using collagen fibre biomaterials. Acta Biomater 2011; 7: 3237-3247

23. O'Brien FJ, Harley BA, Yannas IV, Gibson LJ. The effect of pore size on cell adhesion in collagen-GAG scaffolds. Biomaterials 2005; 26: 433-441

24. Noyes FR, Grood ES. The strength of the anterior cruciate ligament in humans and Rhesus monkeys. J Bone Joint Surg Am 1976; 58: 1074-1082

25. Gentleman E, Livesay G, Dee K, Nauman, E. Development of Ligament-Like Structural Organization and Properties in Cell-Seeded Collagen Scaffolds in vitro. Ann Biomed Eng 2006; 34: 726-736

26. Wang M-C, Pins GD, Silver FH. Collagen fibres with improved strength for the repair of soft tissue injuries. Biomaterials 1994; 15: 507-12

27. Gentleman E, Lay AN, Dickerson DA, Nauman EA, Livesay GA, Dee KC. Mechanical characterization of collagen fibers and scaffolds for tissue engineering. Biomaterials 2003; 24: 3805-3813

28. Kato YP, Dunn MG, Zawadsky JP, Tria AJ, Silver FH. Regeneration of Achilles tendon with a collagen tendon prosthesis. Results of a one-year implantation study. J Bone Joint Surg Am 1991; 73: 561-574

29. Aurora A, McCarron J, Ianotti JP, Derwin K. Commercially available extracellular matrix materials for rotator cuff repairs: State of the art and future trends. J Shoulder Elb Surg 2007; 16: S171-S178

30. Chen J, Xu J, Zheng M. Scaffolds for tendon and ligament repair: review of the efficacy of commercial products. Expert Rev Med Devices 2009; 6: 61-73

31. Friess W, Lee G. Basic thermoanalytical studies of insoluble collagen matrices. Biomaterials 1996; 17: 2289-2294

32. Miles CA, Avery NC, Rodin VV, Bailet AJ. The Increase in Denaturation Temperature Following Cross-linking of Collagen is Caused by Dehydration of the Fibres. J Mol Biol 2005; 346: 551-556

33. Cavallaro JF, Kemp PD, Kraus KH. Collagen fabrics as biomaterials. Biotechnol Bioeng 1994; 43: 781-791

34. Sung NH. Structure and Properties of Collagen and Gelatine in the Hydrated and Anhydrous State. [PhD]. Boston: M.I.T.; 1972 115-. 
35. Itoi E, Berglund LJ, Grabowski JJ, Schultz FM, Growney ES, Morrey BF, An KN.Tensile Properties of the Supraspinatus Tendon. J Orthop Res. 1995 Jul;13(4):578-84. 